stellung gelangen. Eine Bemerkung zu der historischen Einleitung: Wenn man die Ausführungen $\mathrm{P}$ e r r i e $\mathbf{r}$ s im ersten Band der Publikationen der neuen französischen Gradmessung in Ekuador liest, die sich mit der Gesehichte der Gradmessung in Peru befassen und wenn man dabei erfährt, mit welcher Zerfahrenheit und Ziellosigkeit gearbeitet wurde, wie die ganze Ausrüstung der Expedition gar nicht den Verhältnissen angepaßt war, wie ferner die beiden Chefs $\mathrm{B}$ ou g $\mathrm{u}$ e $\mathrm{r}$ und $\mathrm{L}$ a $\mathrm{Con}$ d a $\mathrm{m}$ in $\theta$ sich so zerstritten haben, daß sie nur mehr schriftlich miteinander verkehrten und nicht einmal gleichzeitig zurückkehrten, so geht es wohl nicht an, diese Gradmessung als eine besonders schöne und gute Arbeit zu preisen. Im Gegenteil, man muß sich wundern, daß dabei überhaupt etwas herausgekommen ist.

\title{
A. Prey.
}

A. Haas, Kleiner Grundriß der theoretischen Physik. W. de Gruyter \& Co., Berlin und Leipzig 1934. Preis geb. RM 5,30.

Der bekannte Verfasser hat etwa in der Art der Göschenschen Bändichen eine kurzgefaßte Einführung in die Grundlagen und die wichtigsten Rechenmethoden der theoretischen Physik geschrieben, die im wesentlichen einen für Chemiker und Naturhistoriker geeigneten Auszug aus seinem bekannten zweibändigen Lehrbuch der Theoretischen Physik darstellt und dessen einzelne Kapitel mit dem dem Verfasser eigenen Geschick ausgewählt wurden.

Herbert Schober.

A. Haas, Physik für jedermann. Verständliche Wissenschaft. J. Springer, Berlin 1933. Preis geb. RM 6,80.

Das Bïchlein dient dazu, einen Liaien in jene Kapitel der Physik einzuführen, die von besonderem Technischen und daher auch allgemeinerem Interesse sind. Hieher gehören: Physikalische Fragen der Photographie, des Rundfunks, des Fernsehens, der Röntgenstrahlen usw., um nur einiges aus der reichen Auswahl zu nennen. In den Text sind mehrere Bilder, insbesondere Photographien des Wiener Großsenders, Ultrarotphotographien usw. eingestreut. Inhalt und Schreibweise sind von der bei Haas gewöhnten anregenden Form.

Herbert Schober.

A. Haas, Materiewellen und Quantenmechanik. Vierte und fünite Auflage, 299 S. Akademische Verlagsgesellschaft m. b. H., Leipzig 1934. Preis geb RM 7,80.

Das beliebte Büchlein ist in neuer wesentlich vermehrter Auflage erschienen. Dureh Hinzufügung eigener Kapitel über die neuesten Ergebnisse der Forschung, insbesondere die infolge der Entdeckung des positiven Elektrons so interessant gewordene Diracsche Theorie sowie eine wesentliche Umarbeitung des Kapitels über die F e rmistatistik ist der Umfang um beinahe 100 Seiten gewachsen.

Die gewohnt flüssige und fesselnde Schreibweise des Verfassers wurde durch eine genaue Durchsicht des Textez noch weiter verbessert; das Werk wird sicher in seiner neuen Auflage jedem Leser große Freude bereiten. Im übrigen sei anf die Besprechung der dritten Auflage in Band 37, Seite 45 der Literaturberichte dieser Zeitschrift verwiesen.

Herbert Schober.

P. Debye, Magnetismus. (Leipziger Vorträge 1933.) S. Hirzel, Leipzig 1933. Preis kart. RM 6,-.

Die bekannten Leipziger Vorträge erstrecken sich diesmal auf das Gebiet des Magnetismus. Die Beiträge stammen von $K$ a $p$ i t $z$ a (The change of resistance of metals in magnetic fields), von G e rl a c h (Zusammenhänge zwischen Magnetisierung und elektrischem Widerstand ferromagnetischer Körper), von Sa ck (Die Beeinflussung der inneren Reibung von $\dot{\mathrm{O}}_{2}$ durch ein Magnetfeld), von $\mathrm{Fr}$ i s ch und St e rn (Uber die magnetische Ablenkung von Wasserstoffmolekülen und das magnetigche Moment des Protons), von K r a m ers (Paramagnetische Eigenschaften der Kristalle seltener Erden), von De $\mathrm{H}$ a as (Supraleiter im Magnetfeld), von Bethe (Theorie des Ferromagnetismus), von Becker (Die technische Magnetisierungskurve) und von Ga ns (Zur Energetik ferromagnetischer Stoffe).

Die angeführten Forschernamen zeigen, daß es wieder gelungen ist, die besten Fachleute für die Vorträge zu gewinnen und außerdem so ziemlich alle Grundfragen der gegenwärtigen Magnetik zu behandeln. Wie auch der Herausgeber bemerkte, wurde möglichst wenig an den einzelnen Vorträgen bei der Zusammenfassung geändert, um den Eindruck der Ursprünglichkeit nicht zu verwischen. 